\title{
Surgical Strategy in Large Vestibular Schwannoma
}

\author{
Georgios Klironomos ${ }^{1}$ Amir Reza Dehdashti \\ ${ }^{1}$ Department of Neurosurgery, North Shore University Hospital at \\ Manhasset, Manhasset, New York, United States \\ 2 Department of Neurosurgery, North Shore University Hospital, \\ Zucker Medical School at Hofstra, Manhasset, New York, \\ United States
}

\begin{abstract}
Address for correspondence Georgios Klironomos, MD, PhD, Department of Neurosurgery, North Shore University Hospital at Manhasset, 300 Community Drive, Manhasset, NY 11030-3876, United States (e-mail: geklironomos@gmail.com).
\end{abstract}

J Neurol Surg B 2019;80(suppl S3):S272-S273.

\begin{abstract}
Keywords

- vestibular schwannoma

- near total resection

- semisitting position

Particular care to facial nerve function preservation should be the ultimate goal in surgery for large vestibular schwannomas. We present a 60 -year-old patient who presented with an enlarging right vestibular schwannoma and nonserviceable hearing. The patient was operated in the semisitting position after a patent foramen ovale was ruled out. During the positioning, the feet were positioned at the level of the heart. Precordial Doppler was used to monitor for air embolism. Straight skin incision and retrosigmoid craniotomy was performed. Specific attention to venous bleeding was made during the approach. Meticulous arachnoid dissection of the capsule preserving the arachnoid plane at the surface of the brain stem and the facial nerve can be achieved more efficiently with the patient in the semisiting position and with bimanual microdissection technique. After drilling of the internal auditory canal (IAC), we were able to achieve near total removal of the tumor, leaving a tiny tumor carpet due to extreme adherence to the nerve. Water-tight dura closure and replacement of the bone flap was performed. The patient woke up with a House-Brackmann grade III facial weakness which improved to grade I at 6 weeks postoperatively. Postoperative magnetic resonance imaging (MRI) showed a tiny residual at the surface of the facial nerve at the entrance of the IAC. Near total (>98\%) resection of large vestibular schwannomas is an acceptable surgical strategy with excellent facial nerve outcome. With appropriate patient positioning in semisitting and proper anesthesiological and surgical management, the risk of air embolism is negligible.

The link to video can be found at: https://youtu.be/ErG9VexbiGw.
\end{abstract}

Conflict of Interest

None.

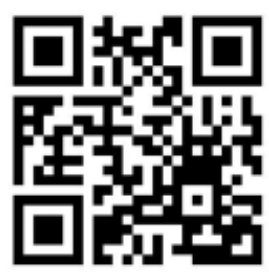

received

July 2, 2018

accepted after revision

August 18, 2018

published online

November 21, 2018

www.thieme.com/skullbasevideos

www.thieme.com/jnlsbvideos

DOI https://doi.org/

10.1055/s-0038-1673702.

ISSN 2193-6331. (c) 2019 Georg Thieme Verlag KG Stuttgart · New York
License terms

(c) $(1) \risingdotseq$ 


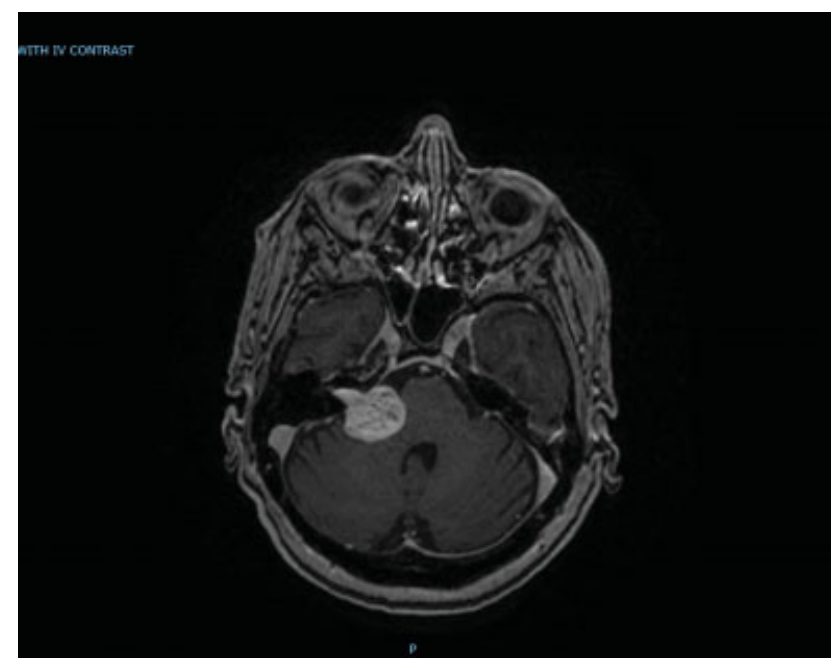

Fig. 1 Axial MRI + gad showing vestibular schwannoma.MRI, magnetic resonance imaging. gad, gadolinium.
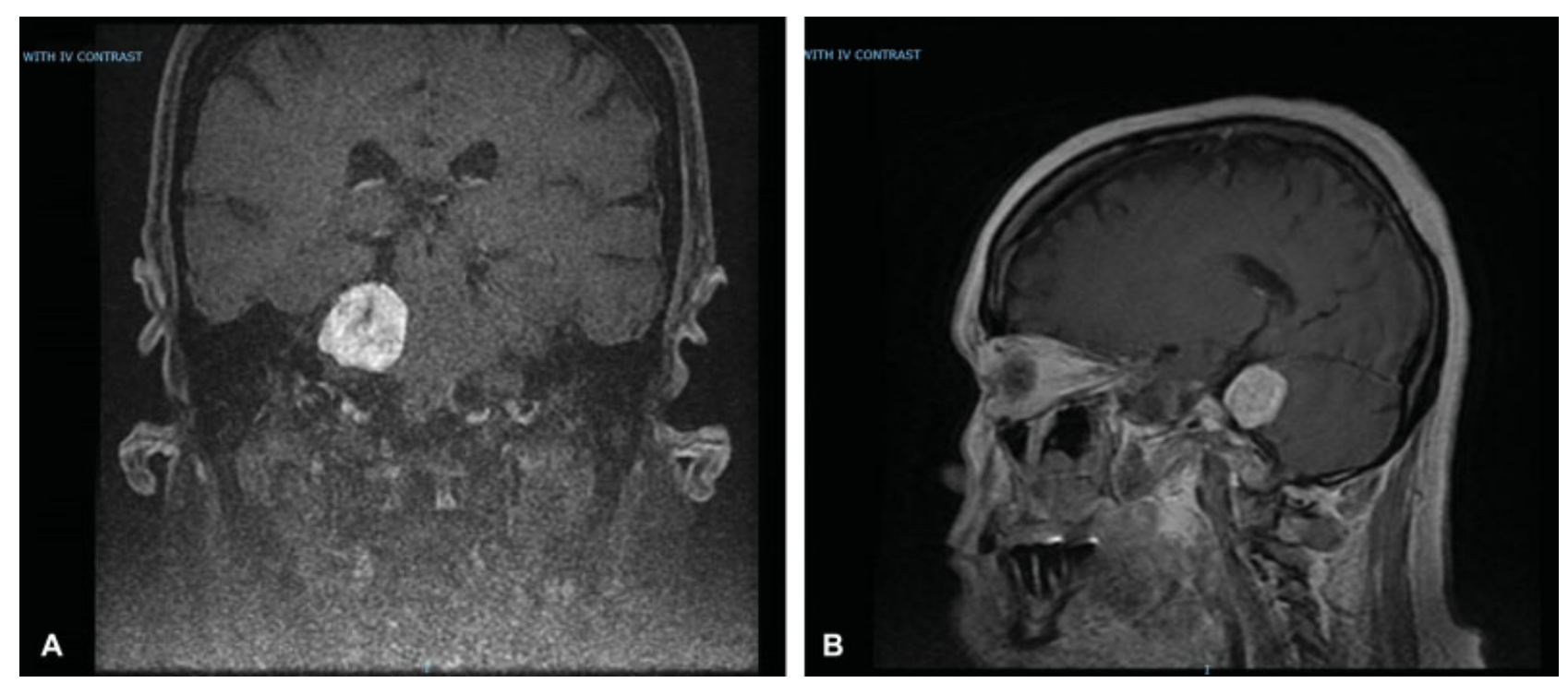

Fig. 2 (A) Coronal MRI + gad. (B) Sagittal MRI + gad. MRI, magnetic resonance imaging. 